\title{
Improved Efficiency of ZnO and Ge Purification
}

\author{
Grygoriy S. Pekar*, Alexandr F. Singaevsky, Myhailo M. Lokshin, Ilya A. Vasin, \\ Oleksandr V. Dubikovskyi \\ V. Lashkaryov Institute of Semiconductor Physics, National Academy of Sciences of Ukraine, Nauki, Kyiv, Ukraine \\ Email: ${ }^{*}$ pekar@isp.kiev.ua, gpekar1@gmail.com
}

How to cite this paper: Pekar, G.S., Singaevsky, A.F., Lokshin, M.M., Vasin, I.A. and Dubikovskyi, O.V. (2019) Improved Efficiency of $\mathrm{ZnO}$ and $\mathrm{Ge}$ Purification. Journal of Crystallization Process and Technology, 9, 39-47.

https://doi.org/10.4236/jcpt.2019.93003

Received: June 25, 2019

Accepted: July 28, 2019

Published: July 31, 2019

Copyright (c) 2019 by author(s) and Scientific Research Publishing Inc. This work is licensed under the Creative Commons Attribution International License (CC BY 4.0).

http://creativecommons.org/licenses/by/4.0/

\begin{abstract}
Unconventional ways to improve the efficiency of purification of two different semiconductor materials of current interest, $\mathrm{ZnO}$ and $\mathrm{Ge}$, are described. It is shown that, by using chemically assisted vapour transport of $\mathrm{ZnO}$ with carbon as a transporting agent, the degree of chemical purity of $\mathrm{ZnO}$ can be increased by more than an order of magnitude. It is also found that heating of the molten $\mathrm{Ge}$ in the experimentally determined narrow (about $20^{\circ} \mathrm{C}$ wide) temperature range in which an intense evaporation of certain substances is observed, leads to a significant reduction of germanium contamination. As a result, a subsequent deep purification of pre-heat treated germanium by zone refining can be achieved at twice reduced (as compared with "non-treated" $\mathrm{Ge})$ number of passes of a boat with germanium through the melting zones. Thus, the Ge purification process becomes faster, cheaper and more efficient.
\end{abstract}

\section{Keywords}

Purification, Germanium, Zinc Oxide

\section{Introduction}

As the areas of application of semiconductor materials expand and requirements to their characteristics increase, the problem of improving methods for materials purification remains relevant. In particular, this applies to such widely used materials as $\mathrm{ZnO}$ and $\mathrm{Ge}$, despite the fundamental differences in their properties and applications.

An example of a highly purified semiconductor material is Ge crystals used as high-resolution $\gamma$ and X-ray detector material, which must contain a very low density of electrically active defects as well as of some other types of defects. By the use of highly advanced technologies of purification, the density of impurities $10^{9}-10^{10} \mathrm{~cm}^{-3}$ is achievable [1]. 
In the case of compound semiconductors, the achievable degree of purification is much less. As to $\mathrm{ZnO}$, for the purification of which the chemical methods are most widely used (see, for example, [2]), $6 \mathrm{~N} \mathrm{ZnO}$ powder is almost the purest material that can be easily found in the market [3].

At the same time, recently the scope of applications of high purity semiconductor materials has expanded. One of the areas of their use is medicine. As known, germanium may be used in the treatment of diseases of the heart and blood vessel, including high blood pressure and high cholesterol; of the eye diseases, including glaucoma and cataracts; of the liver diseases, including hepatitis and cirrhosis. Ge promotes the transfer of oxygen in the tissues of the body (like hemoglobin), enhances the human immune status, promotes the increase of antitumor activity, increases resistance to viruses and bacteria, promotes removal of toxins from tissues, increases frequency of conduction of nerve impulses. In its turn, $\mathrm{ZnO}$ is used in medicine as an antiseptic, a drying, astringent, absorbent substance. It is added to many external dermatological products for the treatment of eczema, pressure sores, baby prickly heat, herpes simplex, wounds, cuts, burns, ulcers. The potential of zinc oxide for antimicrobial applications in the fields of biomedicine and environmental protection was also established [4]. For most of these applications, it is desirable to use fairly pure substances. Except for medical purposes, it might be profitable to use high purity $\mathrm{ZnO}$ crystals for some technical applications, for example, in electronics, primarily as substrates for GaN light-emitting diodes and lasers instead of hydrothermally grown $\mathrm{ZnO}$ crystals contaminated with impurities during growth.

This article describes unconventional, fairly simple and affordable ways for increasing efficiency of $\mathrm{ZnO}$ purification as well as of Ge zone-refining.

\section{Zinc Oxide Purification}

For zinc oxide powder purification, we used a method of chemically assisted vapour transport (CVT). The essence of this method is that the initial material (for example, powder) and the transporting agent (solid or gaseous) are placed in a closed ampoule, both ends of which are heated to different temperatures. In some conditions, a reaction occurs between the initial material and the transporting agent in the hot zone of the ampoule, the reaction products move into the cold zone and there the reverse chemical reaction occurs resulting in formation of the crystallites of the initial material and a gaseous transporting agent. The crystallites settle on the inner walls of the ampoule, and the transporting gas is transferred back to the hot zone and there reacts again with the initial material remaining in this zone. This process may be repeated until the complete transfer of the initial material to the crystallization zone. The advantage of this process, when it is used for growing crystals, is that it takes place at much lower temperatures compared to crystal growing by sublimation followed by vapour transfer from the hot zone to the cold zone with subsequent crystallization there.

It is also known that, in addition to crystal growing, CVT may be used for pu- 
rification of some metals, for example $\mathrm{Zr}, \mathrm{Ni}, \mathrm{Ti}$, etc., using iodine and other transporting agents. At the same time, when using CVT for growing crystals of II-VI compounds, to which $\mathrm{ZnO}$ belongs, the grown crystals (such as $\mathrm{CdS}$, etc.) turned out often to be heavily doped with atoms of the transporting agent. Because of this, CVT seemed inapplicable for the purification of II-VI compounds.

At the same time, the encouraging results could be also found in the literature regarding the possibility of growing sufficiently pure $\mathrm{ZnO}$ crystals by CVT method. In particular, when using chloride or carbon as transporting agents, the residual concentration of chlorine in the crystals was really high (about $0.05 \%$ ), but the residual carbon was found only on the surface of the grown crystals [5]. In addition, when transporting agent $\mathrm{CO}$ was used, the level of carbon contamination in the crystals was not dramatically high, at least in comparison with the impurity content in hydrothermally grown $\mathrm{ZnO}$ crystals [6].

Summarizing known, including the above, information, it can be concluded that preliminary predictions regarding the possibility of $\mathrm{ZnO}$ refining by the CVT process may be doubtful. There are a number of facts against this possibility. Firstly, $\mathrm{ZnO}$ may be contaminated by the transporting agent, secondly, the purified material cannot be CVT-refined from elements-analogs, whose presence in the material is difficult to establish previously at their low concentration, and, thirdly, the volatile substances, if present, can transfer to the cold zone even without the participation of a transporting agent and can contaminate the purified material. Thus, the final conclusion about the possibility of material purification by CVT method can be made only as a result of experiments.

To clarify the possibility of $\mathrm{ZnO}$ purification by CVT, we used a vertical scheme of CVT growing. About $100 \mathrm{~g}$ of $\mathrm{ZnO}$ powder of a purity $99.9 \%$ and a small amount of carbon powder were placed at the bottom of the sealed quartz glass ampoule mounted inside the vertical furnace. As our experiments showed, the amount of carbon powder used did not significantly affect the experimental results and could be, for example, $0.5 \%$ - $2 \%$ of the weight of $\mathrm{ZnO}$ powder. The ampoule with $\mathrm{ZnO}$ and carbon powders was kept at a temperature of about $1000^{\circ} \mathrm{C}$ for about 30 hours. As a result, about $75 \mathrm{~g}$ of $\mathrm{ZnO}$ was crystallized in a cold zone of the ampoule in the form of a sintered fine crystalline powder.

To compare the chemical purity of the initial and recrystallized powders, their mass spectrometric analysis was performed. The studies were made on an Ion-TofIV time-of-flight mass spectrometer. All samples were studied under the same conditions, those were the pressure about $10^{-9} \mathrm{mbar}$, the use of the source of $\mathrm{Bi}_{3}$ ions at an energy of $25 \mathrm{keV}$ and a raster of $41 \times 41 \mu \mathrm{m}$. The surface of the samples was cleaned for $60 \mathrm{sec}$. by the oxygen ions at energy of $1 \mathrm{keV}$ and a raster of $700 \times 700 \mu \mathrm{m}$. When carrying out the experiments, accumulation of the same signal of the main isotope $\mathrm{Zn}$ was made.

Figures 1(a)-(c) show the intensities of the signals of the main contaminants, which were potassium, sodium and the transporting agent, i.e. carbon. As seen, the content of these impurities after refining was reduced from 2 to 60 


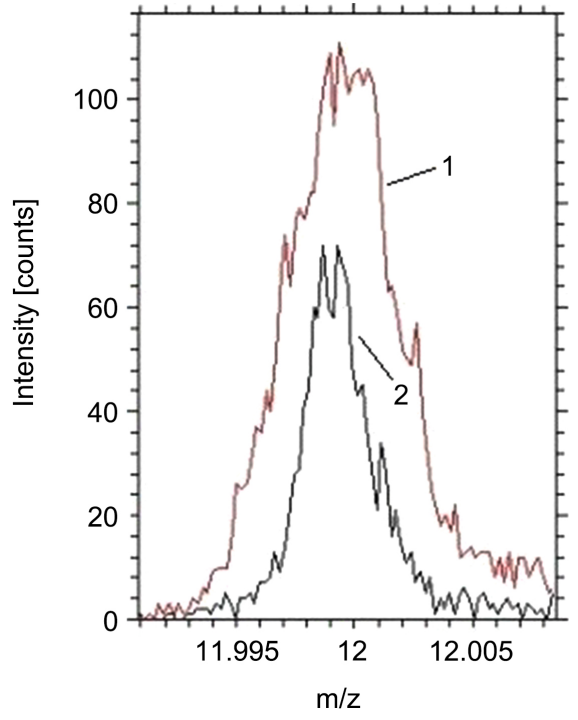

(a)

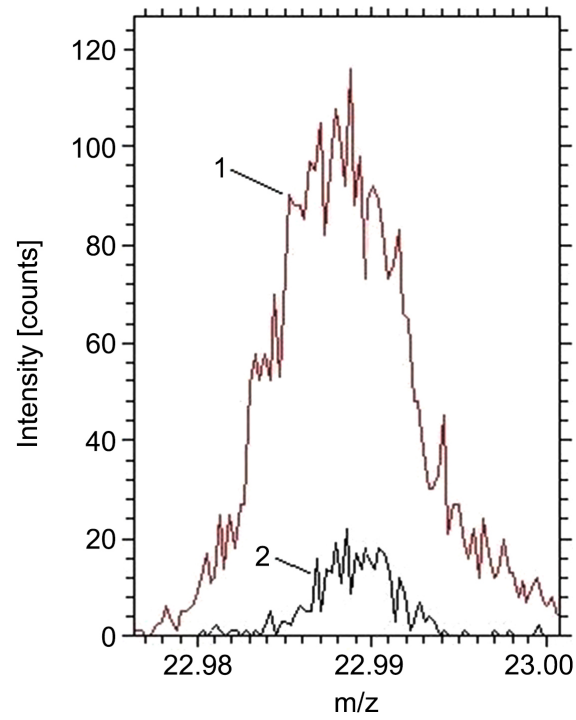

(b)

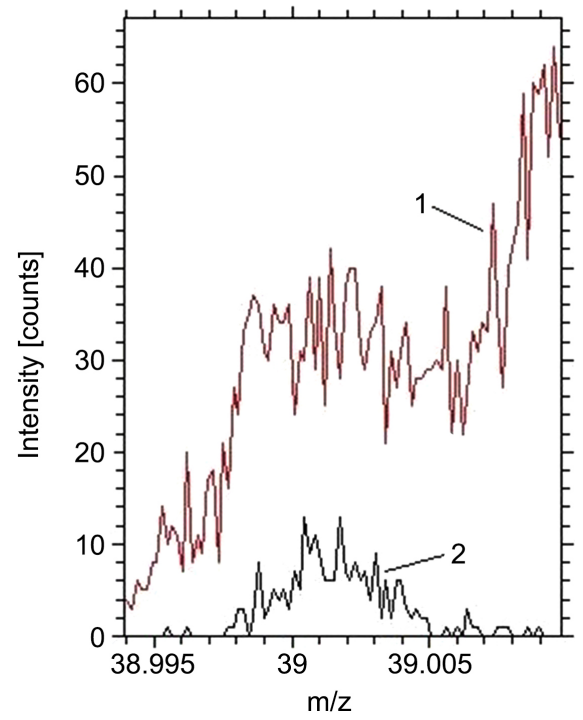

(c)

Figure 1. Mass-spectra of carbon (a), sodium (b) and potassium (c) atoms in the initial (curves 1) and recrystallized (curves 2 ) $\mathrm{ZnO}$ powders.

times. The analysis of the total content of impurities in the initial and purified materials showed that it decreases from $2 \times 10^{19} \mathrm{~cm}^{-3}$ to $5 \times 10^{17} \mathrm{~cm}^{-3}$, respectively, that is, more than by an order of magnitude.

Mass-spectra of constituent elements (zinc and oxygen atoms) as well as of $\mathrm{Zn}_{2}$ and $\mathrm{ZnO}$ molecules in the initial (curves 1) and recrystallized (curves 2) $\mathrm{ZnO}$ powders are shown in Figures $2(\mathrm{a})-(\mathrm{d})$. As can be seen, the signal intensities of the elements that make up the crystal lattice of the sample became higher after recrystallization due to a decrease in the concentration of contaminants.

We also attempted to achieve a purification effect using other transporting agents, such as $\mathrm{CO}$ and $\mathrm{H}_{2} \mathrm{O}$, as well as other evaporation and crystallization temperatures. However, all these experiments were unsuccessful. 


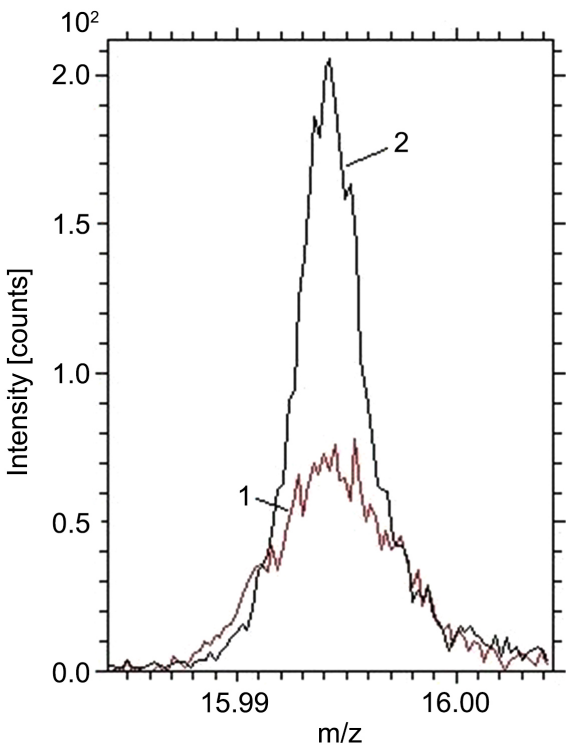

(a)

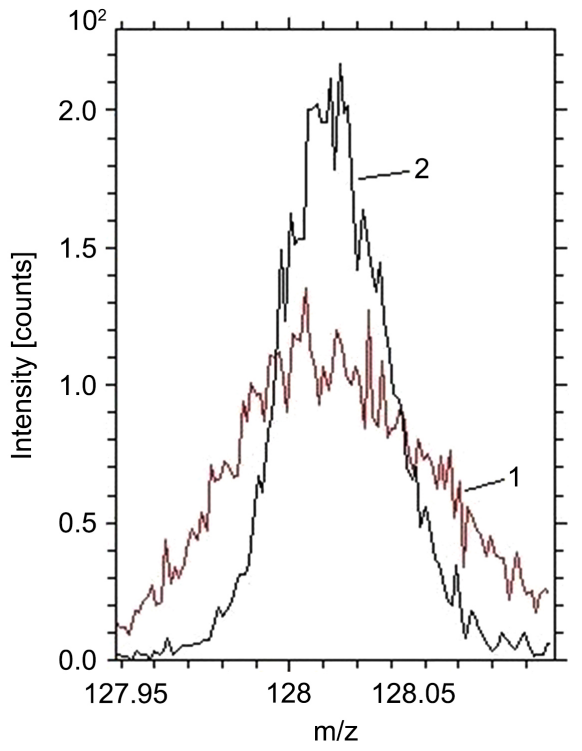

(c)

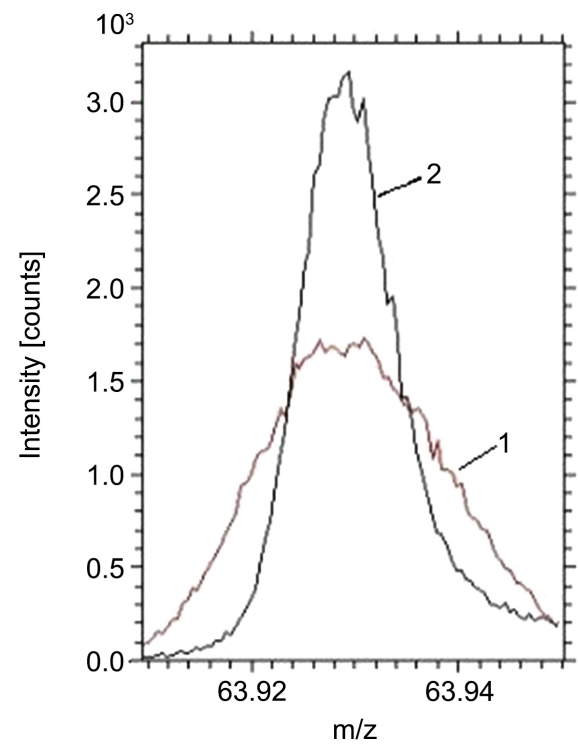

(b)

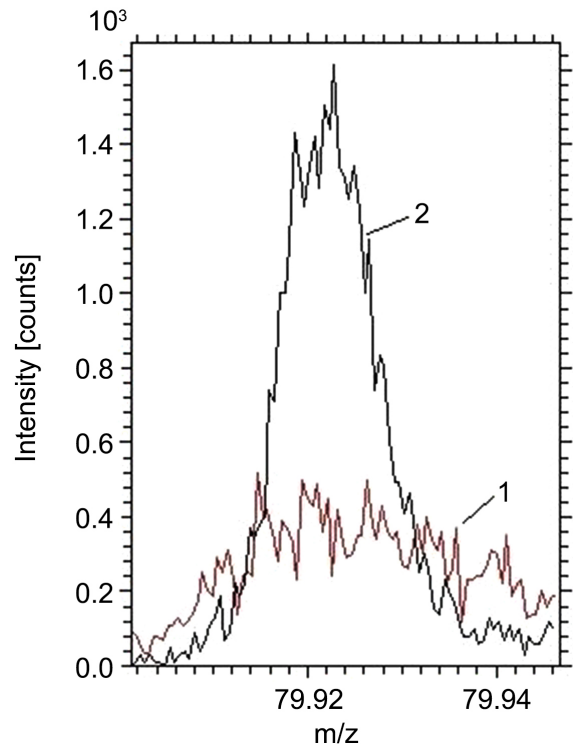

(d)

Figure 2. Mass-spectra of constituent elements and molecules in the initial (curves 1) and recrystallized (curves 2) $\mathrm{ZnO}$ powders: oxygen atoms (a), zinc atoms (b), $\mathrm{Zn}_{2}$ molecules (c) and $\mathrm{ZnO}$ molecules (d).

Note that the question still remains how deep purification can be achieved in the described way, for example, by repeatedly CVT purifications of the same material. We hope to receive an answer to this question in future studies.

\section{Germanium Purification}

As known, germanium is usually successfully refined by zone melting which makes it possible to prepare material with a total density of uncontrolled impurities $10^{9}-10^{10} \mathrm{~cm}^{-3}$. Since $1 \mathrm{~cm}^{3}$ of germanium contains $4.4 \times 10^{22}$ atoms, it can be easily estimated that zone-refined Ge contains only 1 impurity atom per $10^{11}$ - 
$10^{12}$ atoms of the host lattice. This makes Ge one of the most chemically pure materials known. It seems doubtful that it will be possible to improve significantly the zone-refining method itself or to develop an alternative method for deeper purification of germanium. Zone-refining method has been used for many years and is based on the fundamental properties of germanium, namely, on the fact that the extinction coefficient of all impurities (except boron) in germanium is less than unity and therefore the solubility of these impurities in liquid germanium is higher than in solid germanium. Therefore, when pulling a narrow molten zone along a germanium ingot, the impurities pass from the solid ingot to the melt and are gradually removed to the end of the ingot.

Despite the apparent simplicity of this method, a fairly large price has to be paid for Ge purification. Really, each Ge ingot must be subjected to multifold purification, i.e. the graphite boat with germanium should be many times and rather slowly pulled through zones of Ge melting. This process is quite long, laborious and energy consuming. However, it is obvious that its effectiveness can be increased by using the purest possible initial material. The unexpected and easily reachable method of preliminary Ge purification is described below.

A commonly used method for converting prefabricated germanium powder to crystalline germanium prior to its zone-refining, is to melt the Ge powder and then to cool it. In our experiments, the Ge powder obtained by reducing germanium oxide in a hydrogen flow at a temperature about $650^{\circ} \mathrm{C}-675^{\circ} \mathrm{C}$, weighing about $2 \mathrm{~kg}$, was placed in a round graphite crucible and then was heated under a pressure of about $10^{-3}$ atm in a continuously pumped closed chamber. The maximum heating temperature was $1050^{\circ} \mathrm{C}-1150^{\circ} \mathrm{C}$, i.e. slightly above the $\mathrm{Ge}$ melting point $\left(938.2^{\circ} \mathrm{C}\right)$ for reliable melting of the entire germanium in the crucible. During heating, we found that a quite narrow region existed, up to $20^{\circ} \mathrm{C}$ wide, in which a rather unexpected process took place that looked like boiling of the melt. Obviously, this could not be the germanium melt boiling, since Ge boiling temperature is much higher $\left(2833^{\circ} \mathrm{C}\right)$. We decided to stop further heating of the furnace immediately after the appearance of "boiling" and to continue further heating only after its completion. It turned out that "boiling" lasted from 5 to $10 \mathrm{~min}$. The temperature of "boiling" was different for different Ge portions and depended, in particular, on Ge nature and initial purity.

Most likely, the described process was due to decomposition and evaporation of some chemical substances of unknown nature. It seems natural to suggest that such substances were initially contained in the germanium dioxide, from which the metal germanium was obtained.

Figure 3 shows the distribution of electrical resistivity, measured at room temperature, along the diameter of the germanium disk heat-treated as described above. Curve 1 represents the resistivity distribution in the case when the heating of the furnace was not stopped after the start of "boiling", and curve 2 presents the case when this stop was carried out. As seen, "the stop of boiling" results in increasing the Ge resistivity from $1-2 \mathrm{Ohm} \cdot \mathrm{cm}$ to $16-18 \mathrm{Ohm} \cdot \mathrm{cm}$. 


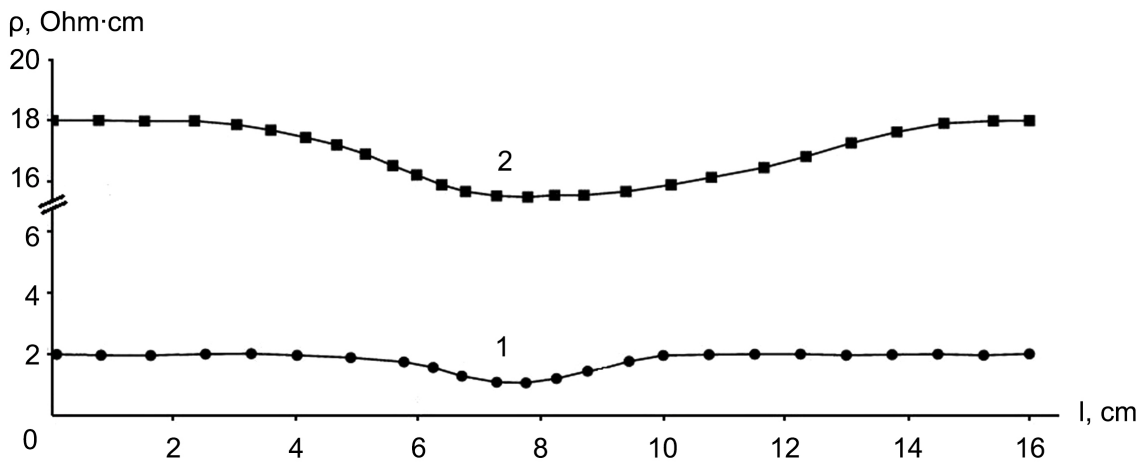

Figure 3. Distribution of electrical resistivity along the diameter of the germanium disk. Curve 1 represents the case when the heating of the furnace was not stopped after the start of "boiling", and curve 2 presents the case when this stop was carried out. Measurements were made at room temperature.

The corresponding values of impurity concentrations were estimated by using the previously established [7] and subsequently repeatedly confirmed experimentally quantitative relationship between the specific resistance of germanium and the density of electrically active impurities. It turned out that that the described above heating process results in the decrease of the density of electrically active impurities from $8 \times 10^{14}-1 \times 10^{15} \mathrm{~cm}^{-3}$ to $7 \times 10^{13}-1 \times 10^{14} \mathrm{~cm}^{-3}$, i.e. by about 10 times.

Let's comment on the fact clearly visible in Figure 3, that the resistivity in the central part of the disk is always slightly less than at the edges of the disk. The reason is that after turning off the furnace heater, the disk is cooled first at the edges, and then in the middle. Therefore, the crystallization front moves from the edges of the disk to the center. As a result, the impurities, like at zone refining, pass from solid Ge to the molten Ge and, hence, gradually displaced into the central region of the disk. It is obvious that such uneven distribution of impurities across the disk has no effect on their total content.

It must be emphasized that the process described above is fundamentally different from the widely used for many decades refining by melting powdered materials in an open crucible installed in a furnace [8]. During remelting, moisture, gases and oxides can be removed from the purified material. However, in this case, the processes of removing impurities occur over a wide temperature range and, hence, no processes similar to boiling in a narrow temperature range can be observed. Therefore it does not make it possible to achieve a deeper purification from impurities of a certain (and unknown) nature, which is achieved by fixing heating in a narrow temperature range.

We used the process described above as preliminary germanium purification before its final zone-refining. It was found that, when using preliminary purified germanium as a material for subsequent zone-refining, the resistivity of almost the entire germanium ingot achieved $47 \mathrm{Ohm} \cdot \mathrm{cm}$ at room temperature after 6 passes of the melted zone. This value of resistivity corresponds to the intrinsic electrical resistivity of germanium [9] and is indicative of its high purity. At the 
same time, the process of zone-refining of the same germanium, which was not subjected to such preliminary purification, resulted in obtaining ingots with a resistivity about $47 \mathrm{Ohm} \cdot \mathrm{cm}$ only in the front half of each ingot even after 12 -fold passage of the melted zone. As for the rest parts of the ingots, their resistivity, as a rule, did not achieve this value even after the multifold zone-refining.

Thus, the developed method of preliminary germanium purification made it possible to increase significantly the efficiency and profitability of the final zone-melting purification.

We have succeeded to apply the described technique, on a fairly significant scale, for preparing germanium ingots used as an initial material when growing optical germanium crystalline plates for thermal imaging systems [10]. To convert zone-refined germanium to so-called optical germanium which is characterized with a high degree of transmission in the infrared region of the spectrum, germanium was doped with $\mathrm{Na}$ so that the free electron densities in the doped crystals were from $5 \times 10^{13}$ to $4 \times 10^{14} \mathrm{~cm}^{-3}$ [11]. The smaller of these values was no less than 1 - 2 orders of magnitude higher than the concentration of free carriers due to uncontrolled impurities in the zone-refined initial germanium used. This difference provided a high degree of reproducibility of the optical properties of germanium resulting from $\mathrm{Na}$ doping. It is obvious that the high efficiency of manufacturing the initial germanium with required parameters described in this article was of utmost importance.

\section{Conclusions}

In the present work, using as an example such materials with quite different properties as $\mathrm{ZnO}$ and $\mathrm{Ge}$, the prospects are shown for searching new, even unconventional, methods for semiconductor materials purification.

Two examples of successful results of such searches are described. It is shown that, by using chemically assisted vapour transport of $\mathrm{ZnO}$ with carbon as a transporting agent, the degree of chemical purity of $\mathrm{ZnO}$ can be increased by more than an order of magnitude. It is also found that heating of the melted metallic germanium carried out in the experimentally determined narrow (about $20^{\circ} \mathrm{C}$ wide) temperature range in which an intense evaporation of substances of unknown nature is observed, results in a decrease, by about 10 times, in the concentration of contaminants in germanium which leads to a large increase in the efficiency of the subsequent final purification of this material by zone-refining.

\section{Acknowledgements}

Financial support from the National Academy of Sciences of Ukraine is acknowledged.

\section{Conflicts of Interest}

The authors declare no conflicts of interest regarding the publication of this paper. 


\section{References}

[1] Fourches, N. and Zielińska, M. (2018) High Purity Germanium: From Gamma-Ray Detection to Dark Matter Subterranean Detectors.

https://hal.archives-ouvertes.fr/hal-01925666 https://doi.org/10.5772/intechopen.82864

[2] Myerson, A.S. and Robinson, P. (1998) Method for the Further Purification of Zinc Oxide. United States Patent No. 5759503.

[3] https://www.americanelements.com/zinc-oxide-powder-1314-13-2

[4] Pathak, T.K., Kroon, R.E., Craciun, V., Popa, M., Chifiriuc, M.C. and Swart, H.C. (2019) Influence of Ag, Au and Pd Noble Metals Doping on Structural, Optical and Antimicrobial Properties of Zinc Oxide and Titanium Dioxide Nanomaterials. Heliyon, 5, e01333. https://doi.org/10.1016/j.heliyon.2019.e01333

[5] Ntep, J.-M., Said Hassani, S., Lusson, A., Tromson-Carli, A., Ballutaud, D., Didier, G. and Triboulet, R. (1999) ZnO Growth by Chemical Vapour Transport. Journal of Crystal Growth, 207, 30-34. https://doi.org/10.1016/S0022-0248(99)00363-2

[6] Santailler, J.-L., Audoin, C., Chichignoud, G., Obrecht, R., Kaouache, B., Marotel, P., Pelenc, D., Brochen, S., Merlin, J., Bisotto, I., Granier, C., Feuillet, G. and Levy, F. (2010) Chemically Assisted Vapour Transport for Bulk ZnO Crystal Growth. Journal of Crystal Growth, 312, 3417-3424. https://doi.org/10.1016/j.jcrysgro.2010.08.046

[7] Prince, M.B. (1953) Drift Mobilities in Semiconductors. Physical Review Journals Archive, 92, 681-687. https://doi.org/10.1103/PhysRev.92.681

[8] Lark-Horovitz, K. and Johnson, V.A. (1959) Preparation and Purification of Materials. In: Methods in Experimental Physics, Vol. 6, Part A, pp. 21-186. Part of Volume: Solid State Physics: Preparation, Structure, Mechanical and Thermal Properties. https://doi.org/10.1016/S0076-695X(08)60453-6

[9] Smith, R.A. (1978) Semiconductors. 2nd Edition, Cambridge University Press, Cambridge, p. 540, Chapter13.

[10] Pekar, G.S., Singaevsky, A.F., Lokshin, M.M., Gordienko, V.I. and Mazurin, I.V. (2018) Large Polycrystalline Optical Germanium Ge:Na Plates with Improved Optical Parameters and Their Application. Semiconductor Physics, Quantum Electronics and Optoelectronics, 21, 173-179. https://doi.org/10.15407/spqeo21.02.173

[11] Pekar, G.S. and Singaevsky, A.F. (2012) Na-Doped Optical Germanium Bulk Crystals. Applied Physics A, 108, 657-664. https://doi.org/10.1007/s00339-012-6947-x 